\title{
Blind individuals show pseudoneglect in bisecting numerical intervals
}

\author{
Zaira Cattaneo • Micaela Fantino • Juha Silvanto • \\ Carla Tinti · Tomaso Vecchi
}

Published online: 2 February 2011

(C) Psychonomic Society, Inc. 2011

\begin{abstract}
Neurologically normal individuals typically show a leftward bias - known as pseudoneglect - in bisecting physical lines as well as numerical intervals. The latter bias may reflect the spatial nature in which numbers are represented (i.e., the mental number line). In previous studies, we found that congenitally blind individuals show such leftward bias in haptic bisection. Here, we demonstrate that blind individuals also show a consistent leftward bias in numerical bisection. The leftward bias was greater when numbers were presented in descending rather than ascending order, and the magnitude of the leftward bias was comparable to that shown by a control group of blindfolded sighted participants. Our findings thus support the view that pseudoneglect operates at a mental representational level rather than being perceptually based. Moreover, the consistent leftward bias shown by blind individuals in both line and numerical bisection suggests that the right hemisphere dominance in spatial processing, resulting in
\end{abstract}

Z. Cattaneo $(\bowtie)$

Department of Psychology, University of Milano-Bicocca,

Piazza dell'Ateneo Nuovo,

Milano, Italy

e-mail: zaira.cattaneo@unimib.it

M. Fantino $\cdot$ T. Vecchi

Department of Psychology, University of Pavia,

Pavia, Italy

\section{J. Silvanto}

Brain Research Unit, Low Temperature Laboratory, Helsinki University of Technology,

Espoo, Finland

C. Tinti

Department of Psychology, University of Torino,

Torino, Italy an overestimation of the left side of space, develops even in the absence of any visual input.

Keywords Mental number line $\cdot$ Blindness $\cdot$ Spatial representation $\cdot$ Numerical cognition $\cdot$ Pseudoneglect

Numerical magnitude is believed to be represented along a mental number line (see Dehaene, Bossini, \& Giraux, 1993). Converging evidence from both neurologically intact individuals and neglect patients has shown that the representation of numbers along the mental number line shares functional properties with the representation of peripersonal and extrapersonal space (see de Hevia, Vallar, \& Girelli, 2008; Umiltà, Priftis, \& Zorzi, 2009).

A major finding in favour of this view is the presence of the same directional bias in the bisection of physical lines and numerical intervals (or corresponding "landmark" tasks) (e.g., Göbel, Calabria, Farnè, \& Rossetti, 2006; Loftus, Nicholls, Mattingley, \& Bradshaw, 2008; Loftus, Nicholls, Mattingley, Chapman, \& Bradshaw, 2009; Longo \& Lourenco, 2007; Oliveri et al., 2004). Specifically, neurologically intact individuals usually tend to bisect to the left of the veridical midpoint in both tasks, a behavioural phenomenon known as "pseudoneglect" (see Jewell \& McCourt, 2000, for a review). In contrast, patients with right-parietal damage resulting in visual neglect consistently show a rightward bias in both line and numerical bisection (e.g., Loftus et al., 2009; Schenkenberg, Bradford, \& Ajax, 1980; Vallar, 2001; Vuilleumier, Ortigue, \& Brugger, 2004; Zorzi, Priftis, \& Umiltà, 2002; see also Pia, Corazzini, Folegatti, Gindri, \& Cauda, 2009, for a consistent leftward bias in both tasks in a neglect patient with left-parietal damage). 
Blindness affects the way spatial information is mentally represented (e.g., Afonso et al., 2010; Noordzij, Zuidhoek, \& Postma, 2006, 2007, for experimental evidence; see Cattaneo, Vecchi, et al., 2008; Thinus-Blanc \& Gaunet, 1997, for reviews). Nonetheless, previous studies have demonstrated that blind individuals are likely to represent numbers in the same spatial format as sighted individuals do-that is, in the form of the mental number line (Castronovo \& Seron, 2007). We have recently demonstrated that totally early blind individuals also tend to err leftward when haptically bisecting wooden rods, suggesting that pseudoneglect in this task does not depend on normal visual experience (Cattaneo et al., in press; Cattaneo, Fantino, Tinti, Silvanto, \& Vecchi, 2010; for previous evidence, see Bradshaw, Nettleton, \& Wilson, 1986; Coudereau, Gueguen, Pratte, \& Sampaio, 2006; Sampaio, Gouarir, \& Mvondo, 1995). However, whether blind individuals also show pseudoneglect in bisecting numerical intervals has not been investigated.

Here, we addressed this issue by testing a group of congenitally blind individuals in a numerical bisection task. In line with evidence obtained with normally sighted individuals (see, e.g., Loftus et al., 2009; Longo \& Lourenco, 2007), one might expect blind individuals to show a consistent leftward bias in bisecting numerical intervals. However, recent evidence has suggested that normal vision may play a different role in the directional bias that emerges in line and numerical bisection tasks: In particular, it has been shown that individuals affected by amblyopia, a developmental disorder affecting spatial vision that results in poor uniocular vision, show a "minineglect" (rightward bias) in line bisection (Thiel \& Sireteanu, 2009) but no significant bias in numerical bisection (Mohr, Mues, Robol, \& Sireteanu, 2010). Whether a similar dissociation exists in totally blind individuals is therefore an empirical question.

\section{Experiment 1}

\section{Method}

Participants Fourteen totally blind subjects ( 8 males), with a mean age of 36.4 years $(S D=7.4$; age range $=23-48$, years of education $=14.4, S D=3.10$ ), took part in the experiment. In no participant was blindness associated with a central neural disorder. Participants were all congenitally blind and were all proficient Braille readers (see Table 1 for details). All participants were right-handed as assessed by the Edinburgh Handedness Inventory (Oldfield, 1971).

Stimuli and procedure The experimental task was adapted from a previous study by Göbel et al. (2006). Partic- ipants were presented aurally with 50 different pairs of three-digit numbers, in ascending order, from "117-166" to "959-984." Each pair of three-digit numbers had the same hundreds digit. The numerical distance between each number pair was either $16,25,36,49$, or 64 . The smaller number was always presented first (see also Göbel et al., 2006; Mohr et al., 2010). Each number pair was used only once for each participant. The number pair stimuli, recorded as a single sound file, were spoken by a female Italian voice. The duration of each sound file was $3.5 \mathrm{~s}$. The verbal stimuli were presented by a computer positioned exactly in front of the participants and aligned with their body's midline. Participants had received instructions to judge and orally report the numerical midpoint of the number pair immediately after the presentation of each stimulus, without calculating. A 3-s time limit was imposed, in order to prevent participants from calculating the middle value. The end of the 3-s response period was signalled with a beep. Responses made after the 3-s period or falling outside the given numerical interval were excluded from statistical analyses. E-Prime (Psychology Software Tools, Pittsburgh, PA) was used to present the stimuli. A series of 10 trials were carried out for each of the five numerical intervals, for a total of 50 trials. Five practice trials were given before starting the experiment. Participants received no feedback as to their performance. The entire experiment lasted approximately $30 \mathrm{~min}$.

\section{Results}

A total of two trials (corresponding to the $0.29 \%$ of the total number of trials) were excluded for responses given after $3 \mathrm{~s}$. The participants' responses were analyzed in terms of how much their judgments of the numerical midpoint of the number pair deviated from the actual midpoint. Deviations from the veridical midpoint were computed by subtracting half of the true size of the numerical interval from the interval between the first given number of the pair and the participant's estimated midpoint (see Göbel et al., 2006; Mohr et al., 2010). This scoring method yields a positive score if the participant's response is to the right of the objective midpoint (in this paradigm, a number greater than the objective centre) and a negative score if the response is to the left of it (a number smaller than the objective centre). Figure 1 shows the participants' mean error as a function of the interval size. A one-sample $t$ test was first carried out comparing the mean bisection error with the null set (zero - that is, the true midpoint). The test was significant, $t(13)=2.31, p=.038$, indicating that, overall, blind participants tended to bisect to the left of the veridical midpoint $(M=1.07$ digits, $S D=1.74)$. 
Table 1 Characteristics of the 18 blind participants who took part in Experiment $1(N=14)$ and Experiment $2(N=10)$

\begin{tabular}{llllll}
\hline Sex & Age & Education (years) & Job Activity & Blindness Aetiology & Onset \\
\hline F & 35 & 10 & Call operator & Optic nerve damage & Birth \\
M & 23 & 16 & University student & Optic nerve damage & Birth \\
F & 36 & 16 & Call operator & Congenital glaucoma & Birth \\
F & 34 & 16 & Call operator & Optic nerve damage & Birth \\
M & 46 & 16 & Call operator & Optic nerve damage (light perception) \\
F & 29 & 18 & English teacher & Retrolental fibroplasias & Birth \\
M & 48 & 13 & Call operator & Optic nerve damage & Birth \\
M & 39 & 10 & Call operator & Optic nerve damage & Birth \\
F & 39 & 13 & Physiotherapist & Optic nerve damage & Birth \\
M & 26 & 18 & Employee & Oxygen therapy & Birth \\
M & 37 & 18 & Call operator & Optic nerve damage & Birth \\
M & 32 & 16 & Employee & Trauma & Birth \\
M & 40 & 11 & Physiotherapist & Oxygen therapy & Birth \\
M & 46 & 10 & Call operator & Optic nerve damage & Birth \\
M & 51 & 13 & Physiotherapist & Oxygen therapy & Birth \\
F & 55 & 17 & Employee & Panophthalmia (light perception until age 16) & Birth \\
F & 59 & 10 & Call operator & Optic nerve damage (light perception) & Birth \\
F & 36 & 16 & Call operator & Optic nerve damage & \\
\hline
\end{tabular}

All participants were proficient Braille readers. Participants had no light perception unless specified.

A one-way repeated measures ANOVA with Size of the Numerical Interval $(16,25,36,49$, or 64$)$ as a within-subjects factor revealed a significant effect of interval size on numerical bisection, $F(4,52)=2.69, p=.041$. Post-hoc $t$ tests (Bonferroni correction applied) did not show any significant difference between any possible pair of intervals; however, the mean bias tended to increase as a function of interval size (see Fig. 1) for intervals 16, 25, 36, and 49, whereas it decreased for the largest interval (64), replicating previous findings (Göbel et al., 2006).

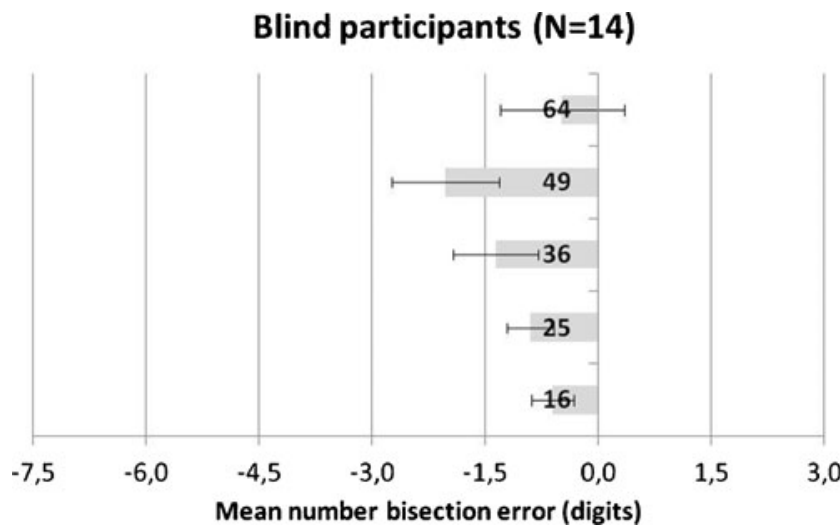

Fig. 1 Participants' mean number bisection error (in digits) for each numerical interval size $(16,25,36,49$, or 64$)$ in Experiment 1 , in which numerical intervals were presented in ascending order only $(N=14)$. Error bars depict \pm SEMs

\section{Experiment 2}

In some of the available studies on numerical bisection, numbers were presented in ascending order only (see Göbel et al., 2006; Mohr et al., 2010). In fact, whereas the order of presentation of the numbers seems not to affect the direction of the bisection bias (Doricchi, Guariglia, Gasparini, \& Tomaiuolo, 2005; Longo \& Lourenco, 2007; Pia et al., 2009), presenting numbers in ascending versus descending order may modulate the magnitude of the bisection bias, with the leftward bias being larger for numbers presented in descending order (see Loftus et al., 2009; Longo \& Lourenco, 2007). To verify whether this is also the case for blind individuals, we carried out a second experiment in which a group of blind participants underwent the same task as in Experiment 1, but with numerical pairs presented in both ascending and descending order. A group of blindfolded sighted participants were also tested; whereas bisection for the same ascending intervals used in Experiment 1 was tested in sighted individuals by Göbel et al. (2006) and Cattaneo, Fantino, Silvanto, Vallar, and Vecchi (2011), sighted individuals' performance for descending-order presentation had not been tested before using these intervals.

Method

Participants A total of 10 blind participants (5 male; mean age $=42.3$ years, $S D=10.3$; age range $=27-59$, years of 
education $=14.3, S D=2.7$ ) took part in Experiment 2. In no participant was blindness associated with a central neural disorder. Participants were all congenitally blind and were all proficient Braille readers (see Table 1 for details). Six participants had already taken part in Experiment 1. A group of 10 sighted participants ( 5 male), comparable to the blind group in age (mean age $=41.9, S D=9.6$; age range $=28$ 56 ) and education (years of education $=14.1, S D=2.2$ ), were tested as controls. All of the blind and sighted participants were right-handed as assessed by the Edinburgh Handedness Inventory (Oldfield, 1971).

Stimuli and procedure The stimuli and procedure were identical to those used in Experiment 1. However, the numbers pairs were randomly presented in both ascending and descending order. For each interval size, there were two trials for ascending order and two trials for descending order. Sighted participants were blindfolded throughout the entire experiment.

\section{Results}

Data were analysed as in Experiment 1. In the blind group, a total of three trials (corresponding to $1.67 \%$ of the total number of trials) were excluded for responses given after $3 \mathrm{~s}$. No trials were excluded in the sighted group. Figure 2 shows the participants' mean errors as a function of the interval size and of ascending and descending order. A one-sample $t$ test was first carried out comparing the mean bisection error in each group for the two orders with the null set (zero- that is, the true midpoint). In the blind group, the test was significant for both ascending (mean leftward bias $=0.67$ digits, $S D=$ 0.88 ), $t(9)=2.40, p=.040$, and descending order (mean leftward bias $=2.39$ digits, $S D=1.47), t(9)=5.14, p=.001$, indicating that, overall, blind participants tended to bisect to the left of the veridical midpoint, regardless of the order in which numerical pairs were presented. In the sighted group, the same pattern was reported for the ascending order (mean leftward bias $=1.22$ digits, $S D=1.60), t(9)=2.43, p=.038$, and the descending order (mean leftward bias $=2.30$ digits, $S D=1.98), t(9)=3.67, p=.005$.

A repeated measures ANOVA with Size of the Numerical Interval $(16,25,36,49$, or 64$)$ and Order as withinsubjects factors and Group as a between-subjects factor revealed a significant effect of order, $F(1,18)=8.85, p=$ .008 , whereas neither the effect of interval size $(p=.48)$, nor the effect of group $(p=.65)$ was significant. A significant Order x Interval Size interaction was reported, $F(4,72)=4.70, p=.002$; no other interactions were significant. The significant effect of order was due to the leftward bisection bias being overall larger when numbers were presented in descending rather than ascending order.
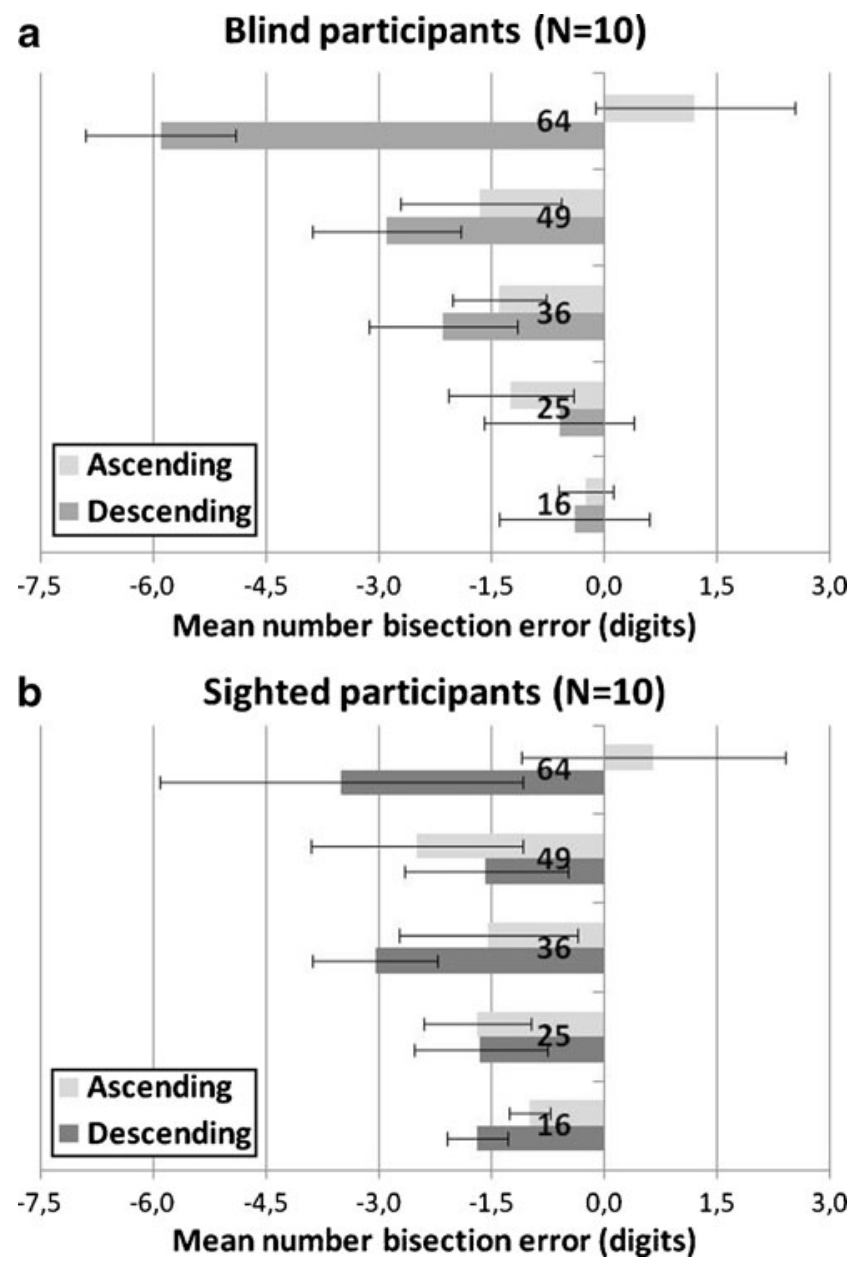

Fig. 2 Mean bisection errors (in digits) for each numerical interval size as a function of order (ascending or descending) in Experiment 2 for (a) blind $(N=10)$ and (b) blindfolded sighted $(N=10)$ participants. Error bars depict $\pm S E M$ s

As shown by Fig. 2, the significant interaction between order and interval size was due to the magnitude of the leftward bias in the descending-order condition increasing proportionally with increases in the interval size, Pearson's $r(100)=.322, p=.001$, whereas this was not the case in the ascending-order condition, Pearson's $r(100)=.12, p=.23$. The lack of an overall significant correlation in the ascending-order condition between the size of the numerical interval and the magnitude of the leftward bias was due to participants' leftward bias reversing to a rightward bias in the interval-64 condition (resembling the pattern of results of Experiment 1; see also Göbel et al., 2006, for a similar finding).

In order to further investigate this phenomenon, standard deviations (i.e., a measure of individual response variability; see Chieffi, Iavarone, \& Carlomagno, 2008) were analysed and subjected to an ANOVA with Numerical Interval Size and Order of Presentation as within-subjects factors and Group as a between-subjects factor (see 
Cattaneo et al., 2011; Cattaneo et al., in press; Chieffi et al., 2008, for a similar analysis). The analysis revealed a significant effect of interval size, $F(4,72)=7.42, p<.001$, indicating that response variability increased linearly with increases in the numerical interval size; the mean variable error was 2.14 for interval 16, 2.65 for interval 25, 3.55 for interval 36, 4.59 for interval 49, and 4.61 for interval 64 . Neither the main effect of order $(p=.70)$ nor the effect of group $(p=.41)$ was significant. None of the interactions were significant.

\section{General discussion}

Congenitally blind individuals were asked to verbally indicate the midpoint of a series of numerical intervals, with the intervals presented either in ascending order (Experiment 1) or in both ascending and descending order (Experiment 2). Blind individuals consistently showed a leftward bias in bisecting numerical intervals, with the magnitude of the leftward bias being comparable to that reported in a matched group of blindfolded sighted participants (Experiment 2). Notably, in both blind and sighted participants, the directional bias was constant (i.e., to the left), regardless of the order (ascending or descending) in which the numerical pairs were presented (see Loftus et al., 2009; Longo \& Lourenco, 2007; Pia et al., 2009).

When the numbers were presented in descending order, the leftward bias was larger overall in both blind and sighted participants, in line with previous evidence (Loftus et al., 2009; Longo \& Lourenco, 2007). Memory-related recency effects (which may have affected the second number if that was used as a reference, enhancing the bias toward the smaller number in the descending-order condition) are unlikely to have played a role, since previous studies (Loftus et al., 2009; Longo \& Lourenco, 2007) found the same effect when the two numbers were simultaneously presented. To account for the greater bias in the descending-order condition, Longo and Lourenco hypothesised that participants may mentally shift the smaller number to the left of the larger one before estimating the midpoint. Such a leftward shift may move attention farther to the left, enhancing the leftward bias. This explanation may also account for the results of our study.

When bisecting ascending intervals, both blind and sighted participants showed a diminished leftward bias (which even turned into a rightward bias in Experiment 2) for the largest interval (64), replicating the result of Göbel et al. (2006) with sighted individuals (see also Cattaneo et al., 2011). This was not the case when bisecting descending intervals: In this condition, the leftward bias became larger as the numerical magnitude increased, being the largest for the interval 64 . The consequence of this was that the overall leftward bias was larger in the descending- than in the ascending-order condition. In one previous study (Cattaneo et al., 2011), in which the same numbers were presented in ascending order only, we hypothesized that such a "crossover effect" depended on response variability (reflecting higher response uncertainty) being the highest in the condition with the largest interval, thus resulting in a less consistent directional bias. However, in the present study, response variabilities for the largest interval were comparable in the ascending- and descending-order conditions; if response variability played a critical role in reversing the direction of the bias for the largest interval, the same effect should have been observed in the descending-order condition, whereas this was not the case. Göbel et al. suggested that the reverse bias observed in bisecting the largest interval (64) may reflect the same mechanisms underlying the effect found by Werth and Poppel (1988) in bisection of imagined lines in healthy participants - that is, a leftward bias for short imagined lines and a rightward bias for long lines (note, however, that this effect has not been consistently replicated in physical line bisection; see Halligan \& Marshall, 1988; McCourt \& Jewell, 1999). In fact, the effect we reported may be interpreted as a sort of "reverse crossover effect," where the crossover effect is a phenomenon usually observed in physical line bisection, according to which neurologically normal individuals tend to err slightly towards the right and neglect patients slightly towards the left when bisecting very short lines $(\sim 2 \mathrm{~cm})$, thus showing the opposite directional bias, as compared to when bisecting longer lines ( 20 cm) (e.g., Monaghan \& Shillcock, 1998, for a review). It has been suggested that this crossover results from the interplay of biases in attentional orientation and magnitude estimation (e.g., Ishiai et al., 2004; Mennemeier et al., 2005). For instance, Ishiai et al. reported that neglect patients tended to generate mental images of visually presented short lines (e.g., $25 \mathrm{~mm}$ ) that were actually longer than their physical length, with a "representational" overextension to the left side. When asked to indicate the midpoint of these imagined lines, patients with neglect placed the subjective midpoint to the right of the centre of the stimulus line for the longer lines (e.g., 100 and $200 \mathrm{~mm}$ ), and to the left for the $25-\mathrm{mm}$ lines. However, the leftward bias in bisecting the shortest lines disappeared when the displacement of the subjective midpoint was measured from the centre of the representational line image (Ishiai et al., 2004). Accordingly, it may be that in our experiment the rightward half of the ascending numerical intervals was somehow overrepresented, so that the rightward bias observed for the largest size (64) was in fact a leftward bias, assuming that the centre of the 
represented interval was shifted to the right of the true midline. However, such an explanation remains speculative, and further experimental evidence needs to be collected before any firm conclusions can be drawn.

We have previously demonstrated (Cattaneo et al., in press; Cattaneo et al., 2010) that congenitally blind individuals tend to err leftward when haptically bisecting physical rods (see also Bradshaw et al., 1986; Coudereau et al., 2006; Sampaio et al., 1995), thus exhibiting pseudoneglect (Jewell \& McCourt, 2000). In sighted participants, the magnitude of the leftward bias in numerical bisection was found to correlate significantly with the magnitude of the leftward bias in physical line bisection (Longo \& Lourenco, 2007). All of the blind participants who took part in Experiment 1 had also been tested in a previous study investigating the existence of representational biases in the vertical, radial, and horizontal planes using a haptic line bisection task (Cattaneo et al., in press). We can thus verify whether the magnitude of the leftward bias correlates between horizontal line bisection and numerical bisection (collapsing across the different interval sizes and line lengths), as seems to be the case in sighted participants (Longo \& Lourenco, 2007). Although the correlation was not significant [Spearman $r(14)=.39, p=.17$ ], when dividing - according to Longo and Lourenco's procedurethe blind participants into "high" and "low" pseudoneglect subgroups on the basis of a median split on their performance on the physical line bisection task (as reported in Cattaneo et al., in press), the leftward bias in bisecting numerical intervals turned out to be greater in the high $(M=$ 1.67 digits) than in the low ( $M=0.48$ digits) pseudoneglect subgroup (although, again, the difference failed to reach significance, $p=.22$ ).

The leftward bias reported in line and numerical bisection may depend on a right-hemisphere dominance in spatial processing, resulting in an overestimation of the left portion of the external space (Kinsbourne, 1970). Blindness does not seem to prevent such hemispheric asymmetry, according to previous studies that have reported a left-field/right-hemisphere superiority in auditory localisation (Gougoux, Zatorre, Lassonde, Voss, \& Lepore, 2005; Voss et al., 2004; Weeks et al., 2000). The fact that such asymmetry is evident in the blind when bisecting numerical intervals, where no perceptual exploratory mechanisms are involved, corroborates the previous evidence that the overestimation of the left side of the mental number line is unaffected by the manipulation of perceptual features of the stimuli, such as the spatial position of the presented numbers or their temporal order of appearance (Loftus et al., 2009). This indicates that pseudoneglect depends on asymmetries in the mental representation of the stimuli rather than being perceptually based (see also Bisiach \& Luzzatti, 1978, for the first evidence of the representational nature of neglect symptoms). Moreover, these findings critically support the view that numbers are represented in the form of a mental number line in blind individuals (Castronovo \& Seron, 2007).

Our results may appear to be at odds with previous evidence from amblyopic individuals who showed a rightward bias ("minineglect") when bisecting physical lines (Thiel \& Sireteanu, 2009) and no consistent directional bias when bisecting numerical intervals (Mohr et al., 2010). It was suggested (see Mohr et al., 2010) that the pattern of performance of amblyopic individuals in bisection tasks may depend on a different organization of the dorsal visual pathway (i.e., the cortical stream that projects from early visual areas to the posterior parietal cortex, and that is involved in space perception and action control; see Milner \& Goodale, 1992) that underlies the representations of both space and numerical magnitude (e.g., Delazer, Karner, Zamarian, Donnemiller, \& Benke, 2006; see Hubbard, Piazza, Pinel, \& Dehaene, 2005, for a review). Indeed, there is evidence that strabismic amblyopia affects the organization of the dorsal visual pathway (see Constantinescu, Schmidt, Watson, \& Hess, 2005; Simmers, Ledgeway, Mansouri, Hutchinson, \& Hess, 2006). Conversely, several neuroimaging studies have found similar patterns of activation in the dorsal visual pathway of totally blind and sighted individuals in tasks involving spatial-attention, working memory, and spatial-imagery processes (e.g., Fiehler, Burke, Bien, Röder, \& Rösler, 2009; Garg, Schwartz, \& Stevens, 2007; Vanlierde, De Volder, Wanet-Defalque, \& Veraart, 2003; but see Collignon, Voss, Lassonde, \& Lepore, 2009). In light of this result, the findings of similar biases in both line and numerical bisection in blind and sighed individuals may be less surprising. Moreover, this adds to behavioural evidence suggesting that the complete lack of vision may be less detrimental for the development of specific representational mechanisms than is monocular blindness (see Cattaneo, Merabet, Bhatt, \& Vecchi, 2008; Vecchi et al., 2006).

We did not find any evidence for a dissociation of blind individuals' performance in numerical bisection and line bisection (Cattaneo et al., in press; Cattaneo et al., 2010). Therefore, our data do not clarify the nature of the dissociation between numerical and line bisection tasks previously reported in a group of right-brain-damaged patients (Doricchi et al., 2005). In particular, it has been hypothesised that numerical bisection involves more frontal cortical circuits (mediating working memory processes), whereas line bisection mainly relies on parietal circuits (Doricchi et al., 2005). It is worth noting that haptic line bisection in the blind (but also haptic bisection in sighted individuals) may require working memory processes to a greater extent than visual bisection does; this is because the line extent needs to be sequentially acquired, requiring a 
continuous updating of the corresponding mental image generated in memory (see Cattaneo et al., in press; Cattaneo et al., 2010). Conversely, a visual line is simultaneously available in all of its extent and does not need to be maintained in memory.

Overall, our data suggest that congenitally blind individuals represent numbers in a spatial format and that they tend to overestimate the left portion of this mental representation, as neurologically intact sighted individuals usually do, possibly reflecting hemispheric asymmetry in spatial processing (Kinsbourne, 1970).

\section{References}

Afonso, A., Blum, A., Katz, B. F., Tarroux, P., Borst, G., \& Denis, M. (2010). Structural properties of spatial representations in blind people: Scanning images constructed from haptic exploration or from locomotion in a 3-D audio virtual environment. Memory \& Cognition, 38, 591-604.

Bisiach, E., \& Luzzatti, C. (1978). Unilateral neglect of representational space. Cortex, 14, 129-133.

Bradshaw, J. L., Nettleton, N. C., \& Wilson, L. (1986). Tactualkinesthesic matching of horizontal extents by the long-term blind: Absence or reversal of normal left side underestimation. Neuropsychologia, 24, 261-264.

Castronovo, J., \& Seron, X. (2007). Semantic numerical representation in blind subjects: The role of vision in the spatial format of the mental number line. The Quarterly Journal of Experimental Psychology, 60, 101-119.

Cattaneo, Z., Fantino, M., Silvanto, J., Vallar, G., \& Vecchi, T. (2011). Tapping effects on numerical bisection. Experimental Brain Research, 1, 21-28.

Cattaneo, Z., Fantino, M., Tinti, C., Pascual-Leone, A., Silvanto, J., \& Vecchi, T. (in press). Spatial biases in peripersonal space in sighted and blind individuals revealed by a haptic line bisection paradigm. Journal of Experimental Psychology: Human Perception and Performance.

Cattaneo, Z., Fantino, M., Tinti, C., Silvanto, J., \& Vecchi, T. (2010). Crossmodal interaction between the mental number line and peripersonal haptic space representation in sighted and blind individuals. Attention, Perception, \& Psychophysics, 72, 885-890.

Cattaneo, Z., Merabet, L. B., Bhatt, E., \& Vecchi, T. (2008). Effects of complete monocular deprivation on visuo-spatial memory. Brain Research Bulletin, 77, 112-116.

Cattaneo, Z., Vecchi, T., Cornoldi, C., Mammarella, I., Bonino, D., Ricciardi, E., \& Pietrini, P. (2008). Imagery and spatial processes in visual impairments. Neuroscience and Biobehavioral Reviews, $32,1346-1360$.

Chieffi, S., Iavarone, A., \& Carlomagno, S. (2008). Effect of spatiotopic factors on bisection of radial lines. Experimental Brain Research, 189, 129-132.

Collignon, O., Voss, P., Lassonde, M., \& Lepore, F. (2009). Crossmodal plasticity for the spatial processing of sounds in visually deprived subjects. Experimental Brain Research, 192, 343-358.

Constantinescu, T., Schmidt, L., Watson, R., \& Hess, R. F. (2005). A residual deficit for global motion processing after acuity recovery in deprivation amblyopia. Investigative Ophthalmology \& Visual Science, 46, 3008-3012.

Coudereau, J. P., Gueguen, N., Pratte, M., \& Sampaio, E. (2006). Tactile precision in right-handed archery experts with visual disabilities: A pseudoneglect effect? Laterality, 11, 170-180. de Hevia, M. D., Vallar, G., \& Girelli, L. (2008). Visualizing numbers in the mind's eye: The role of visuo-spatial processing in numerical abilities. Neuroscience and Biobehavioral Reviews, 32, 1361-1372.

Dehaene, S., Bossini, S., \& Giraux, P. (1993). The mental representation of parity and number magnitude. Journal of Experimental Psychology: General, 122, 371-396.

Delazer, M., Karner, E., Zamarian, L., Donnemiller, E., \& Benke, T. (2006). Number processing in posterior cortical atrophy-A neuropsychological case study. Neuropsychologia, 44, 36-51.

Doricchi, F., Guariglia, P., Gasparini, M., \& Tomaiuolo, F. (2005). Dissociation between physical and mental number line bisection in right hemisphere brain damage. Nature Neuroscience, 8 , $1663-1665$.

Fiehler, K., Burke, M., Bien, S., Röder, B., \& Rösler, F. (2009). The human dorsal action control system develops in the absence of vision. Cerebral Cortex, 19, 1-12.

Garg, A., Schwartz, D., \& Stevens, A. A. (2007). Frontal eye field activity during auditory spatial attention in early blind. Neuropsychologia, 45, 2307-2321.

Göbel, S. M., Calabria, M., Farnè, A., \& Rossetti, Y. (2006). Parietal rTMS distorts the mental number line: Simulating "spatial" neglect in healthy subjects. Neuropsychologia, 44, 860-868.

Gougoux, F., Zatorre, R. J., Lassonde, M., Voss, P., \& Lepore, F. (2005). A functional neuroimaging study of sound localization: Visual cortex activity predicts performance in early-blind individuals. PLoS Biology, 3, e27.

Halligan, P. W., \& Marshall, J. C. (1988). How long is a piece of string? A study of line bisection in a case of visual neglect. Cortex, 24, 321-328.

Hubbard, E. M., Piazza, M., Pinel, P., \& Dehaene, S. (2005). Interactions between number and space in parietal cortex. Nature Reviews. Neuroscience, 6, 435-448.

Ishiai, S., Koyama, Y., Nakano, N., Seki, K., Nishida, Y., \& Hayashi, K. (2004). Image of a line is not shrunk but neglected: Absence of crossover in unilateral spatial neglect. Neuropsychologia, 42, 251-256.

Jewell, G., \& McCourt, M. E. (2000). Pseudoneglect: A review and meta-analysis of performance factors in line bisection tasks. Neuropsychologia, 38, 93-110.

Kinsbourne, M. (1970). A model for the mechanism of unilateral neglect of space. Transactions of the American Neurological Association, 95, 143-146.

Loftus, A. M., Nicholls, M. E. R., Mattingley, J. B., \& Bradshaw, J. L. (2008). Left to right: Representational biases for numbers and the effect of visuomotor adaptation. Cognition, 107, 1048-1058.

Loftus, A. M., Nicholls, M. E., Mattingley, J. B., Chapman, H. L., \& Bradshaw, J. L. (2009). Pseudoneglect for the bisection of mental number lines. The Quarterly Journal of Experimental Psychology, 62, 925-945.

Longo, M. R., \& Lourenco, S. F. (2007). Space perception and body morphology: Extent of near space scales with arm length. Experimental Brain Research, 177, 285-290.

McCourt, M. E., \& Jewell, G. (1999). Visuospatial attention in line bisection: Stimulus modulation of pseudoneglect. Neuropsychologia, 37, 843-855.

Mennemeier, M., Pierce, C. A., Chatterjee, A., Anderson, B., Jewell, G., Dowler, R., Mark, V. (2005). Biases in attentional orientation and magnitude estimation explain crossover: Neglect is a disorder of both. Journal of Cognitive Neuroscience, 17, 1194-1211.

Milner, A. D., \& Goodale, M. A. (1992). Separate visual pathways for perception and action. Trends in Neuroscience, 15, 20-25.

Mohr, H. M., Mues, H. T., Robol, V., \& Sireteanu, R. (2010). Altered mental number line in amblyopia: Reduced pseudoneglect corresponds to a decreased bias in number estimation. Neuropsychologia, 48, 1775-1781. 
Monaghan, P., \& Shillcock, R. (1998). The cross-over effect in unilateral neglect: Modelling detailed data in the line-bisection task. Brain, 121, 907-921.

Noordzij, M. L., Zuidhoek, S., \& Postma, A. (2006). The influence of visual experience on the ability to form spatial mental models based on route and survey descriptions. Cognition, 100, 321342.

Noordzij, M. L., Zuidhoek, S., \& Postma, A. (2007). The influence of visual experience on visual and spatial imagery. Perception, 36, 101-112.

Oldfield, R. C. (1971). The assessment and analysis of handedness: The Edinburgh Inventory. Neuropsychologia, 9, 97-113.

Oliveri, M., Rausei, V., Koch, G., Torriero, S., Turriziani, P., \& Caltagirone, C. (2004). Overestimation of numerical distances in the left side of space. Neurology, 63, 2139-2141.

Pia, L., Corazzini, L., Folegatti, A., Gindri, P., \& Cauda, F. (2009). Mental number line disruption in a right-neglect patient after a left-hemisphere stroke. Brain and Cognition, 69, 81-88.

Sampaio, E., Gouarir, C., \& Mvondo, D. (1995). Tactile and visual bisection tasks by sighted and blind children. Developmental Neuropsychology, 11, 109-127.

Schenkenberg, T., Bradford, D. C., \& Ajax, E. T. (1980). Line bisection and unilateral visual neglect in patients with neurological impairments. Neurology, 30, 509-517.

Simmers, A. J., Ledgeway, T., Mansouri, B., Hutchinson, C. V., \& Hess, R. F. (2006). The extent of the dorsal extra-striate deficit in amblyopia. Vision Research, 46, 2571-2580.

Thiel, A., \& Sireteanu, R. (2009). Strabismic amblyopes show a bilateral rightward bias in a line bisection task: Evidence for a visual attention deficit. Vision Research, 49, 287-294.
Thinus-Blanc, C., \& Gaunet, F. (1997). Representation of space in blind persons: Vision as a spatial sense? Psychological Bulletin, $121,20-42$.

Umiltà, C., Priftis, K., \& Zorzi, M. (2009). The spatial representation of numbers: Evidence from neglect and pseudoneglect. Experimental Brain Research, 192, 561-569.

Vallar, G. (2001). Extrapersonal visual unilateral spatial neglect and its neuroanatomy. Neuroimage, 1, S52-S58.

Vanlierde, A., De Volder, A. G., Wanet-Defalque, M. C., \& Veraart, C. (2003). Occipito-parietal cortex activation during visuo-spatial imagery in early blind humans. Neuroimage, 19, 698-709.

Vecchi, T., Cattaneo, Z., Monegato, M., Pece, A., Cornoldi, C., \& Pietrini, P. (2006). Why Cyclops could not compete with Ulysses: Monocular vision and mental images. NeuroReport, 17, 723-726.

Voss, P., Lassonde, M., Gougoux, F., Fortin, M., Guillemot, J. P., \& Lepore, F. (2004). Early- and late-onset blind individuals show supra-normal auditory abilities in far-space. Current Biology, 14, 1734-1738.

Vuilleumier, P., Ortigue, S., \& Brugger, P. (2004). The number space and neglect. Cortex, 40, 399-410.

Weeks, R., Horwitz, B., Aziz-Sultan, A., Tian, B., Wessinger, C. M., Cohen, L. G., Rauschecker, J. P. (2000). A positron emission tomographic study of auditory localization in the congenitally blind. The Journal of Neuroscience, 20, 2664-2672.

Werth, R., \& Poppel, E. (1988). Compression and lateral shift of mental coordinate systems in a line bisection task. Neuropsychologia, 26, 741-745.

Zorzi, M., Priftis, K., \& Umiltà, C. (2002). Brain damage: Neglect disrupts the mental number line. Nature, 417, 138-139. 